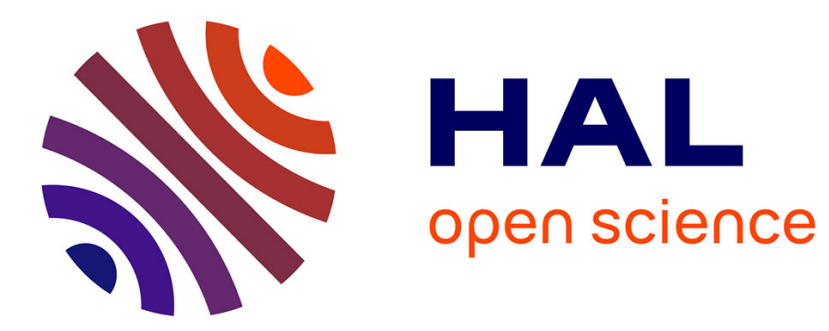

\title{
Un nouveau joint relatif au culte de Lallupiya - Istanuwa (CTH 773)
}

\author{
Alice Mouton
}

\section{To cite this version:}

Alice Mouton. Un nouveau joint relatif au culte de Lallupiya - Istanuwa (CTH 773). Zeitschr. f. Assyriologie Bd., 2008, 98, pp.251-261. halshs-00359805

\section{HAL Id: halshs-00359805 https://shs.hal.science/halshs-00359805}

Submitted on 9 Feb 2009

HAL is a multi-disciplinary open access archive for the deposit and dissemination of scientific research documents, whether they are published or not. The documents may come from teaching and research institutions in France or abroad, or from public or private research centers.
L'archive ouverte pluridisciplinaire HAL, est destinée au dépôt et à la diffusion de documents scientifiques de niveau recherche, publiés ou non, émanant des établissements d'enseignement et de recherche français ou étrangers, des laboratoires publics ou privés. 


\title{
Un nouveau joint relatif au culte de Lallupiya - Ištanuwa (CTH 773) ${ }^{1}$
}

\author{
par Alice Mouton - Strasbourg
}

\begin{abstract}
À partir d'un nouveau joint entre les deux fragments hittites KBo 47.266 et KUB 35.136+, le présent article offre une nouvelle édition de ce texte. Celui-ci décrit une cérémonie religieuse relevant du culte de Lallupiya et Ištanuwa, deux villes de l'Anatolie occidentale. La col. i décrit certains des gestes rituels qui sont effectués lors de la cérémonie alors que la col. iv détaille les paroles des chants louvites entonnés à cette occasion par le chœur des hommes d'Ištanuwa.
\end{abstract}

Alors que je collationnais, en Septembre 2007, les tablettes hittites d'Ankara relatives au culte de Lallupiya et d'Ištanuwa (CTH 773)2 ${ }^{2}$, deux villes de l'Anatolie occidentale, j'ai pu mettre en évidence un nouveau joint direct, que je souhaite présenter ici. Je tiens à exprimer ma gratitude envers M. Hikmet Denizli, directeur du Musée des Civilisations Anatoliennes d'Ankara qui m'a aimablement accueillie lors de mon séjour dans son musée. Je remercie également le Dr. Rukiye Akdoğan pour son aide précieuse. Le joint complète la tablette KUB 35.136+ qu'avait éditée F. Starke en $1985^{3}$. Il classait celle-ci dans la catégorie «Festbeschreibung mit Wechselgesängen» dans le contexte du culte du panthéon d'Ištanuwa ${ }^{4}$. La présence de nombreux chants choraux au sein de la cérémonie religieuse est en effet l'une des principales caractéristiques du culte d'Ištanuwa - Lallupiya5. La tablette KUB 35.136+ est datée NS et plus particulièrement de la fin du XIVème siècle par F. Starke, ce qui correspond à

\footnotetext{
${ }^{1}$ Mes remerciements vont au Pr. Theo van den Hout qui m'a fait l'amitié de relire attentivement cet article. Bien que j’aie suivi au mieux ses précieux conseils, je reste la seule responsable des erreurs qui ont pu subsister. Les abréviations employées ici sont celles se trouvant dans: H. G. Güterbock/H. A. Hoffner (éd.), The Hittite Dictionary of the Oriental Institute of the University of Chicago (= CHD), L-N, Chicago 1989, xxi-xxix; CHD P, Chicago 1997, vii-xxvi; CHD Š, Chicago 2002, vi-viii.

2 Tablettes transcrites par Starke (1985). Ces collations interviennent dans le cadre du projet de recherche intitulé «The religion of the Luwians: A Survey of Religious Practice in Western Anatolia in the Hittite Period», que le Pr. Ian Rutherford (Université de Reading) et moi-même avons initié.

3 Starke (1985, 326-328). Voir également la translittération de Melchert (2001).

4 Starke $(1985,295)$.

5 Pour une synthèse sur le culte d'Ištanuwa - Lallupiya, voir Hutter (2003, 239-243).
} 
sa phase IIIa ${ }^{6}$. Les différents fragments la composant ont tous été mis au jour à Büyükkale dans le bâtiment $\mathrm{Bk} \mathrm{A}$, dans la pièce $\mathrm{n}^{\circ} 2$ pour la plupart d'entre eux?

Le joint direct présenté ici est KBo 47.266 + KUB 35.136 + KBo 29.209. KBo 47.266 (numéro d'inventaire 483/w) est un grand fragment de tablette (env. $12 \mathrm{~cm}$ de long) dont la copie a été publiée en 2005 par H. Otten, Ch. Rüster et G. Wilhelm. Les copistes ont suggéré plusieurs mises en parallèle entre des passages de KBo 47.266 et KUB 35.135, KBo 9.93 et KBo 46.2588. Ma collation indique un joint direct entre le Recto de ce fragment et le haut de KUB 35.136 i (voir fig. 1). Ce joint permet ainsi d'obtenir presque l'intégralité de la colonne i de la tablette: comme l'indiquent à juste titre les copistes, le bord supérieur de la tablette est proche. Outre le fait qu'il complète la col. i, ce joint apporte une autre information importante: il rend à KUB 35.136+ une partie de son colophon (voir fig. 2).

La tablette ainsi reconstituée, on s'aperçoit que sa composition peut être divisée en fonction des colonnes: la col. i décrit les objets nécessaires à la cérémonie religieuse, ainsi que certains des gestes rituels effectués. Quant à la col. iv, elle retranscrit principalement les chants accompagnant la cérémonie. Cette composition bipolaire se retrouve dans d'autres tablettes du même corpus, telles que KUB 35.142 et KUB 35.39, notamment ${ }^{9}$.

Dans la col. i de KBo 47.266+, un grand nombre de denrées alimentaires sont vraisemblablement destinées au repas de la ou des divinité(s) impliquée(s) dans la cérémonie. Outre cela, les principaux acteurs rituels sont nommés: le roi et la reine (i $13^{\prime}$ ). Au moins une séquence rituelle se déroule dans un «champ non cultivé» (gimra-), lieu important pour cette cérémonie, puisqu'il est mentionné dans le colophon fragmentaire du texte (iv $\left.39^{\prime \prime}\right)$. Des tentes, élément récurrent des textes relevant du culte de Lallupiya - Ištanuwa, sont utilisées à plusieurs reprises (i $31^{\prime}, 35^{\prime}$, etc.). La col. i se termine par des offrandes de pains à une divinité.

La col. iv ne décrit plus ni les objets ni les gestes rituels, mais au contraire les chants qui sont entonnés lors de la cérémonie. Généralement, deux chefs de chœur chantent en premier, puis l'ensemble du

${ }^{6}$ Starke $(1985,302$ et 27$)$.

7 Voir le site internet de S. Košak, Konkordanz der hethitischen Texte, http://www.hethport.uni-wuerzburg.de/hetkonk/.

8 KBo 47, VIII.

9 Starke (1985, 323-325, texte II.2. et 328-330, texte II.5). Voir également Starke (1985, 296-297). 
chœur, que le texte appelle «l'assemblée» (panku-), répète les mêmes phrases musicales. Les chants sont en langue louvite, à l'instar des autres témoignages relevant du culte de Lallupiya - Ištanuwa. Leurs paroles sont répétitives: elles sont à la première personne du singulier et font allusion à deux éléments, à savoir un Gišhilu- et un Giš ilu-, qui sont qualifiés de hauts. Étant donné la proposition de H. C. Melchert, de faire de Gišiluun arbre, on pourrait suggérer que Giš hilu- désigne lui aussi un arbre, bien que d'une autre espèce ${ }^{10}$. La fin de la col. iv, juste avant le colophon, semble décrire à nouveau les gestes rituels, et non plus les chants qui les accompagnent (iv $25^{\prime}-30^{\prime}$ ).

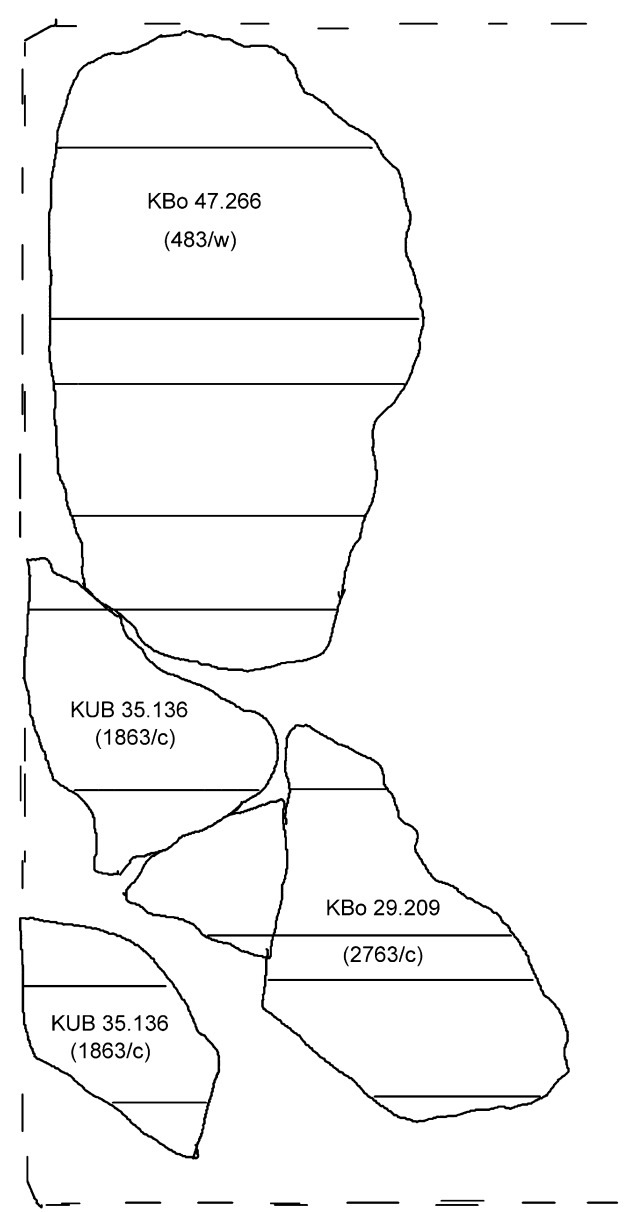

fig. 1: KBo 47.266+ col. i

10 Melchert (1993, 88 et 68$)$. 


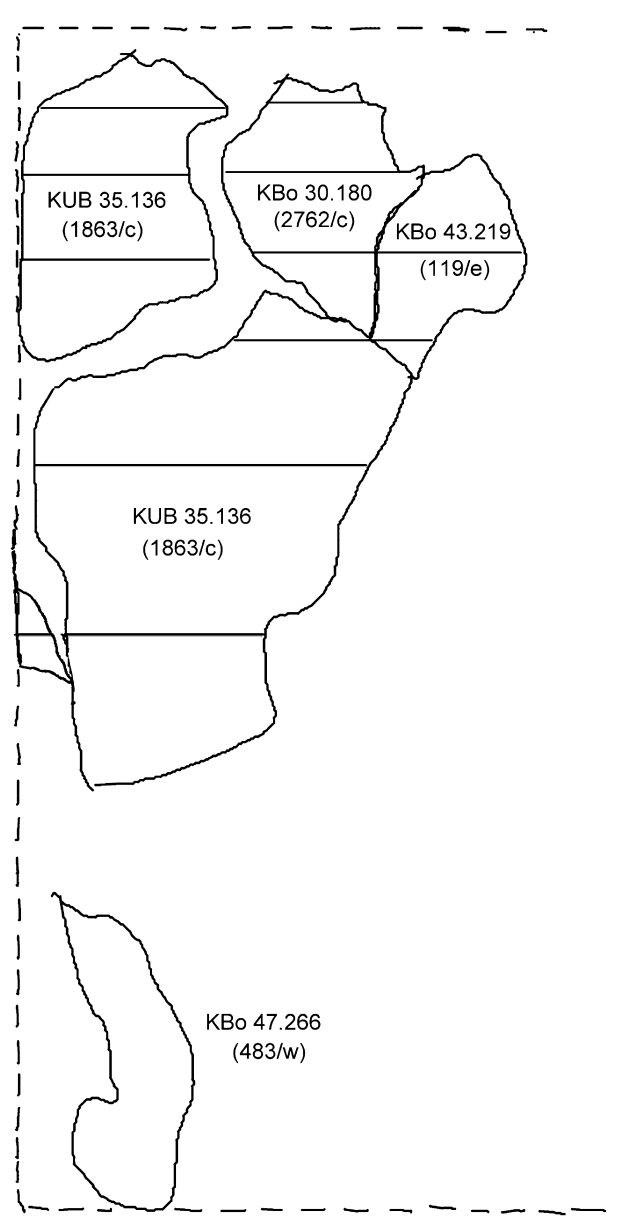

fig. 2: KBo $47.266+$ col. iv

Édition de KBo 47.266+

col. i

$\mathrm{x}+1$. [

$$
\text { ] } \mathrm{T}\left[\mathrm{A}^{?}\right.
$$

$2^{\prime}$. ]DA? [

$3^{\prime}$. $\quad[$ ...]-it $\mathrm{x}[$

$4^{\prime}$. [o] X x [ $\ldots-p]$ a pár-hu- ${ }^{\top} e^{\top}-[n a$

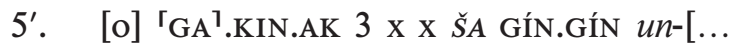

6'. 1 wa-ak-šur İ.DÙG.GA 1 wa-ak-šur İ.UDU x[ 


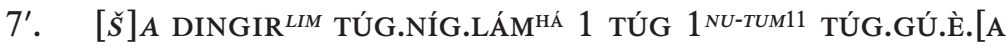

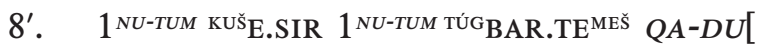

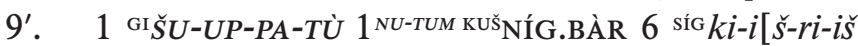

10'. '66121 síg $k i$-iš-ri-iš BABBAR $1{ }^{\text {TÚG }}$ ta-kar-ri-iš ${ }^{13} \mathrm{x}$ [

11'. hu-it-ti-ya-u-aš 1 MA.NA URUDU 1 MA.NA A.B[ÁR?14

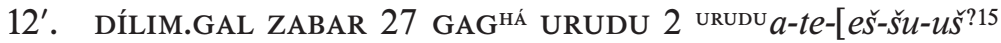

13'. [n] $u$ ma-a-an LUGAL MUNUS.LUGAL a-ni-an-zi nu $2 \times[$

14'. ha-an-da-a-an-zi ma-a-an IT-TI LUGAL MUNUS.L[UGAL

15'. [x]-nu-ma-ši-uš DÙ-an-zi URUDU [ ] x x x x [

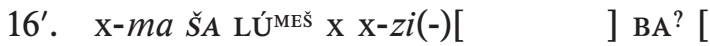

17'. $\mathrm{x}$ TÚG.GÚ.È.A x [o] x [o] x [o] x [ ] ZI? [

18'. [ ]-gi-nu-wa-al-li ta-ha-[o o] $\mathrm{x} \times$ [

\begin{tabular}{|c|c|}
\hline $19^{\prime}$. & 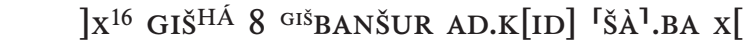 \\
\hline $20^{\prime}$. & ] $4 T A-P A L$ BABBAR $4 T A-P A[L$ \\
\hline $21^{\prime}$ & ]X-mu 2 GIš $A \check{S}-H A-L U M$ AD.[KID $\check{S} A^{17}$ \\
\hline $22^{\prime}$. & ] 4 ŠU.NAG.NAG $3{ }^{\text {GIš }} t i^{1}-\left[i-p a-a \check{s}^{18}\right.$ \\
\hline $23^{\prime}$. & ] 2 GišGU.ZA GÍD.DA 2 Giš[ $[\ldots$ \\
\hline $24^{\prime} . \quad[$ & 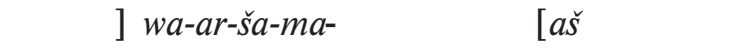 \\
\hline
\end{tabular}

25'. [ ] GIR $4{ }^{\mathrm{HA}} 4{ }^{\mathrm{DUG}}{ }^{\prime} \mathrm{DÍLIM}^{\prime}$ (UD).GAL A x[

26'. [ DUG?]NA-AK-DAM-MU'HÁ [

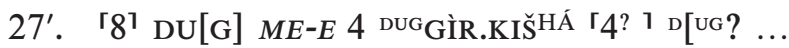

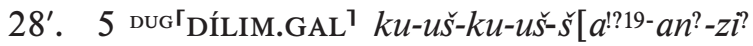

29'. na-aš-ta ma-ah-ha-an ki-i hu-u-m[a-an

11 KUB 35.135 i 8': $1^{\text {NU-TIM }}$.

12 Restitution à partir de la ligne précédente et de KUB 35.135 i 10' qui doit par conséquent être lu: 6 síg $k[i$-iš-ri-iš $]$.

13 Comme cela a déjà été noté par H. Otten, Ch. Rüster et G. Wilhelm (KBo 47, VIII), KUB 35.135 i $11^{\prime}$ indique: 1 rúg $t a-k a ̀ r$ !(AM)-ri-iš.

14 La tablette présente le début de clous horizontaux qui conviendrait au signe BÁR:

15 ateššuš se retrouve dans KBo 29.199 col. droite 7': Starke (1985, 316).

16 Il pourrait s'agir d'un signe UD. Dans ce cas, peut-être faut-il lire: [Ú-NU-U]T.

17 Restitution à partir de KBo 46.258:4'.

18 Restitution à partir de KBo 46.258:6'.

19 Les traces présentes sur la tablette ne sont pas conformes au signe šA attendu dans ce contexte: 
30'. nu a-ra-ah-za 'gi-im'-ri 5 T [A-PAL GIšZA.LAM.GAR ${ }^{20}$

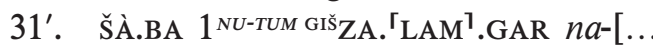

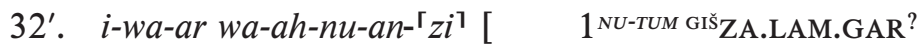

33'. MUNUS.LUGAL-an-na ku-wa-pí an- ${ }^{\Gamma} d a^{1}$ [

34'. [i] š-ki-ya-an-ta DINGIR ${ }^{\text {LUM- }} k a ́ n ~ k u{ }^{-}{ }^{\top} w a-p i^{1}$ [

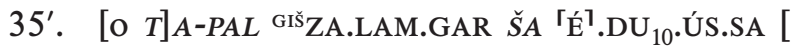

36'. [ku-w]a-pí an-da-an ar-re-e[s]-kán-zi x[

37'. [ ] ]x- $a n^{21}$ GIǏZZA.LAM.GAR ${ }^{\mathrm{HA}-T I M} z i-i n-n a-\left[\ldots{ }^{22}\right.$

38'. [ ] ]x-ša-an-zi 1 GrššÚ.A pár-ku-u ti-an-z[i

39'. [ ] ] nu-uš-ši TÚG.GÚ.Ė.A TÚG.GÚ.È.A [

40'. [ ] ]x-ma-[x]-kán TúG-it an-da ka-a-ri-y[a- ...

41'. [ $\quad \ldots-y] a-m a-w a$ Ú-UL du-uk-ka-a-ri $n[a-\ldots$

42'. [ ] ] nam-ma-aš-ši-kán $\{$ nam-ma-aš-ši-kán $\}{ }^{\mathrm{T}}{ }^{\mathrm{U}} \mathrm{BA}\left[\mathrm{R}^{?}{ }^{2} \mathrm{TE}^{? 23}\right.$

$43^{\prime} . \quad[\quad \ldots-y] a-a \check{s}-\check{s i}$ šar-ku-ya-an- $\quad[\ldots$

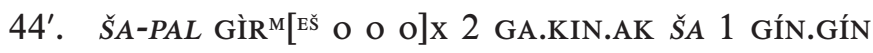

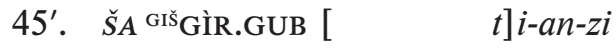

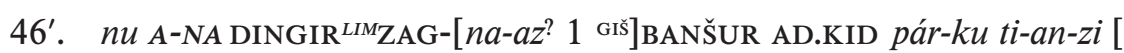

47'. A-NA GIŠBANŠUR-ma-aš-š[a-an o o O NI]NDA.ERÍN.MEŠ da-a-i še-ra$a \check{s}-\check{s} a-a n 1{ }^{\mathrm{NINDA}} w a-[g e-e \check{s}-s ̌ a r$

48'. ku-ra-an-ta da-a-i š[e-ra-aš-š $] a$-an 1 NINDA.SIG 1 NINDA ši-i-lu-haa-an [

49'. NINDA $t u-u z-z i-i n d a-{ }^{\top} a^{1}-[i$ GÙB ?-la]-az-zi-ya-aš-ši 1 GIŠBANŠUR AD.KI[D pár-ku ti-an-zi

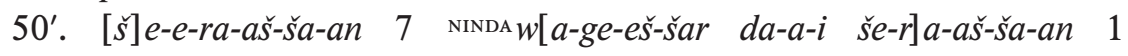
NINDA wa-ge-ě̌-šar $\mathrm{x}[\quad$ da-a-i]

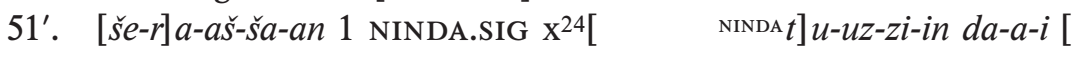

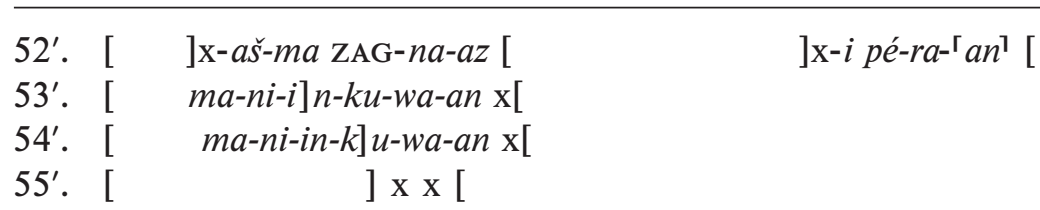

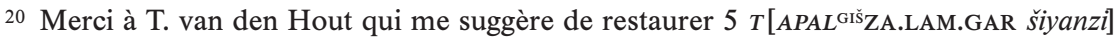
ou quelque chose d'équivalent (courrier électronique du 7. 02. 2008).

${ }^{21}$ On peut suggérer $[m a-a h-h] a-a n$.

22 Sans doute $z i-i n-n a-[a n-z i]$.

${ }^{23}$ Les traces que je vois sur la tablette vont en faveur d'une lecture BAR: $D$ W///.

${ }^{24}$ Un chiffre égal ou supérieur à 4 d'après les traces sur la tablette. 
col. iv

$\mathrm{X}+1$. [DUMUMEš].LUGAL-ya [

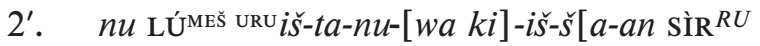

$3^{\prime}$. za-a-am-mu Gišhe-e-[lu-w]a ${ }^{25}$ a-ru-u-wa za-[a-mu i-lu-u-wa a-ruu-wa ${ }^{26}$ ]

4'. zi-i-la a-ru-u-w[a] a-ú-i-ha Dki-i-[na-li- ...

5'. $\quad$ pa-an-ku-ša-ma-aš-[ma $k a t]-t a\langle-a n\rangle \quad Q A^{27}-T A M-M A-p a ́ t \quad a[r-k u-w a-$ $i z-z i]$

6'. nam-ma 2 LÚMEš G[AL-ŠU-N]U ki-iš-ša-an [SÌR]RU[ø]

7'. za-a-mu i-lu-ú-w[a a-ru-ú] $\langle\langle-w a ? 28\rangle\rangle-w a$ za-a-mu Gš̌hi-i-e[1]〈-lu $\rangle-w a$ $\{x\}[a-r u-u ́-w a]$

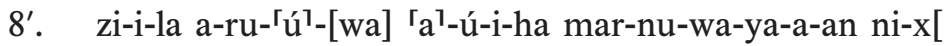

9'. $\quad p a-a n-k u-u ́-s ̌ a-a \check{-}-m[a-a \check{s} \quad k a t]-t a-a n \quad Q A-T A M-M[A-p] a ́ t \quad a r-k u-w a-$ $i[z-z i]$

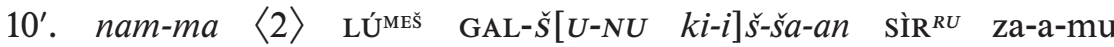
$i ?-1[u ?-u-w a]$

11'. zi-i-la 'a'-[ru-u-wa a-ú-i-ha za-a]-mu Gišhi-i-lu!-wa zi-i-[la a-ru-u-wa]

12'. a-ú-i-ha x[ ]x-iš-mi pa-an-ku-u[š-ma-aš-ma-aś]

13'. $k a t^{-}{ }^{\top} t a^{\top}-[$ an $Q A-T A M-M A-p a ́ t]{ }^{\top} a r^{\top}-k u^{-}{ }^{\top} z i^{\top}[\varnothing]$

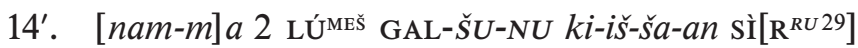

15'. [za-a-m]u GIši-lu-u-wa za-a-am-mu GIšhi-i-lu-[wa zi-i-la]

16'. [a-r]u-u-wa a-ú-i-ha hu-u-tar-la-a-an ni-[...

17'. [pa]-an-ku-uš-ma-aš-ma-aš kat-ta-an QA-TAM-[MA-pát ar-ku-wa$i z-z i]$

18'. [n]am-ma 2 LÚMEš GAL-ك̌U $-N U k i-i \check{s}-\check{s} a-a\left[n\right.$ SÌR $\left.^{R U}\right]$

25 Voir Melchert $(1993,68)$.

26 Restitution hypothétique basée sur iv $7^{\prime}$ et iv $19^{\prime}$.

27 Inscrit sur une érasure.

${ }^{28}$ Lecture du signe incertaine. Les traces semblent indiquer un signe wA à moitié préservé: 亥.

29 Contrairement à ce qu'indique Starke (1985, 327 note 132), les traces sont conformes au début du signe Sìr: 
19'. [za]-a-am-mu Giši-lu-u-wa a-ru-u-wa [za-a-am-mu Gišhi-i-lu-wa a-ru-u-wa $\left.{ }^{30}\right]$

20'. [z]i-i-la a-ru-u-wa a-ú-i-ha [

21'. [pa-a]n-ku-uš-ma-aš-ma-aš kat-ta-a[n QA-TAM-MA-pát ar-ku-wa$i z-z i]$

22'. [o $U M]-M A \operatorname{sìn}^{R U}$ a-ú-i-ha $x[$

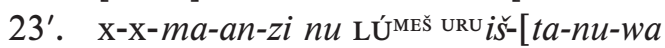

24'. EG[I]R GIǏIG ar-ta $n u \mathrm{x}[$

25'. ' $n a^{1}-[a s ̌]-t a$ LUGAL MUNUS.LUGAL DUMU ${ }^{\mathrm{M}[\mathrm{Es̆}}$.LUGAL

26'. [an-d] a-an pa-a-an-zi nu [

27'. [ ] ] pé-e-di x[

28'. [ a]r-ta nu-za-an [

29'. [ ] ] $-z a$ Ú-UL $\mathrm{x}[$

$30^{\prime} .[\quad \ldots]-y a\{\mathrm{x} x\}[$

(lacune de 5 lignes env.)

$36^{\prime \prime}$. DUB.[...

$37^{\prime \prime} . \check{S} A[$

$38^{\prime \prime}$. DUMUMEั [

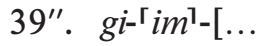

col. i

Les premières lignes sont trop mal préservées pour être traduites.

$4^{\prime}$. [...] du parhuena-,

$5^{\prime} . \quad[\mathrm{x}]$ fromage(s), trois ... $\mathrm{d}^{\prime}$ (un) sicle, [...],

6'. un wakšur d'huile fine, un wakšur de graisse de mouton [...].

7'. Les habits de luxe de la divinité: un vêtement, un manteau [...],

$8^{\prime}$. une paire de chaussures, des vêtements BAR.TE ensemble avec $[\ldots]$,

9'. un roseau, un rideau, six kišri- [de couleur ...],

10'. six kišri- de couleur blanche, un vêtement takarri-, [...]

$11^{\prime}$. du fait de tirer, une mine de cuivre, une mine de $\operatorname{plo}[\mathrm{mb}, \ldots]$

$12^{\prime}$. un grand récipient DílIM en bronze, vingt-sept clous en cuivre, deux haches en cuivre [...].

$13^{\prime}-14^{\prime}$. Quand le roi et la reine célèbrent (cette fête), on apprête deux [...].

30 Restitution hypothétique basée sur les chants louvites précédents. 
$14^{\prime}-15^{\prime}$. Quand avec le roi et la rei[ne,...] on fait ...,

$15^{\prime}$. $\quad[\ldots]$ en cuivre $[\ldots]$.

$16^{\prime} . \quad \ldots$ des hommes ... [...]

$17^{\prime} . \quad \ldots$ manteau $[\ldots]$

$18^{\prime} . \quad \ldots[\ldots]$

$19^{\prime}$. [...] en bois, huit tables en vannerie dont $\mathrm{x}[\ldots]$

20'. [...] quatre de couleur blanche, quatre [de couleur ...]

21'. ..., deux récipients AŠHALUM en vann[erie de ...],

$22^{\prime}$. [...], quatre récipients ŠU.NAG.NAG, trois $t i[p a-]$,

$23^{\prime}$. [...] deux chaises longues, deux [...],

24'. [...] du bois à brûler [...].

$25^{\prime}$. [...] en céramique, quatre grands récipients Dílim [...],

$26^{\prime}$. [...] couvercles $[\ldots]$,

27'. huit récipients d'eau, quatre récipients Gír.KIš, quatre r[écipients ...],

28'. cinq grands récipients Dílim $[o n]$ moud $[\ldots]$.

29'. Quand [on a préparé] tout cela,

$30^{\prime}$. dehors, dans un champ non cultivé, [on monte] cinq [tentes]

$31^{\prime}$. dont une tente [...]

$31^{\prime}-32^{\prime}$. on fait tourner $[\ldots]$ comme $[\ldots]$.

$32^{\prime}-33^{\prime}$. [Une tente] où [l'on ... le roi et] la reine,

$33^{\prime}-34^{\prime}$. [où ....] (sont) oints, où [...] la divinité,

$35^{\prime}-36^{\prime}$. [...] tentes [en guise] de tarnu- où ils se lavent, [...].

37'. [Quand on] termine (d'installer) les tentes,

$38^{\prime}$. on [...]. On place [la divinité sur une chaise haute ${ }^{31}[\ldots]$

$39^{\prime}$. [...] un manteau, un manteau à elle [...]

$40^{\prime}$. [...] (v. couvrir) [...] à l'aide d'un vêtement [...]

$41^{\prime}$. [...] n'est pas prescrit. [...]

$42^{\prime}$. [...] Ensuite, un vêtement BA[R.TE?] à elle [...]

$43^{\prime}$. [...] (v. chausser) à elle $[\ldots]$

44'. Sous les pieds [...] deux fromages d'un sicle

$45^{\prime}$. on place $[\ldots]$ du tabouret.

31 Pour parku- utilisé pour qualifier un meuble, voir CHD P, 161 sub parku- d. 
46'. À droite de la divinité, on place [une] table haute de vannerie [...]

$47^{\prime}-48^{\prime}$. Sur la table, il/elle place du pain de soldats. Par-dessus, il/elle place un pain $w a[$ geššar ...] découpés.

48'-49'. Par-dessus, il/elle place un pain plat, un pain šiluhān, [...], un pain tuzzi.

49'. À sa gauche, [on place] une (seconde) table haute en vannerie $[\ldots]$

50'. Par-dessus, [il/elle place] sept pains $w[$ agěšar $]$. Par-dessus, [il/elle place] un pain wageššar.

51'. Par-dessus, il/elle place un pain plat [...], un pain tuzzi. [...]

$52^{\prime} . \quad$ À droite de $[\ldots],[\ldots]$ face à $[\ldots]$

$53^{\prime}$. $\quad[\ldots$ à pro $]$ ximité $[\ldots]$

$54^{\prime} . \quad[\ldots$ à proxi $]$ mité $[\ldots]$

$55^{\prime} . \quad[\ldots] \ldots[\ldots]$

col. iv

$\mathrm{x}+1$. et [les princes] royaux [...]

2'. Les hommes d'Ištanu[wa chantent a]ins[i]:

3'. «Ces hilu- (sont) hauts pour moi, ces [arbres ilu- (sont) hauts pour moi!]

4'. Donc je suis monté, la divinité Kinaliya [...].»

5'. L'assemblée c[hante] avec eux la même chose [en réponse].

6'. Ensuite, deux de [leu]rs c[hefs chant]ent ainsi:

7'. "Ces arbres ilu- (sont) [hau]ts pour moi, ces hilu- (sont) [hauts] pour moi!

$8^{\prime}$. Donc je suis monté, ... [...]»

9'. L'assemblée chan[te av]ec e[ux] la même cho[se] en réponse.

10'. Ensuite, 〈deux〉 de 1[eurs] chefs chantent [ai]nsi: «Ces arbres $i l[u]$ - (sont hauts) pour moi!

$11^{\prime}-12^{\prime}$. Donc [je suis] mon[té. Ces] hilu- (sont hauts) pour moi! Don $[c]$ je suis $[\mathrm{m}]$ onté. $[\ldots] »$

$12^{\prime}-13^{\prime}$. L'assemblée chante ave[c eux la même chose] en réponse.

14'. [Ensuit]e, deux de leurs chefs chant[ent] ainsi:

15'. «[Ces] arbres ilu- (sont hauts) [po]ur moi, ces hilu- (sont hauts) pour moi! [Donc] 
16'. je suis $[\mathrm{m}]$ onté, $\ldots[\ldots] \gg$

17'. L'[a]ssemblée [chante] avec eux la même cho[se en réponse].

18'. [E]nsuite, deux de leurs chefs [chantent] ains[i]: [...]

19'. «[C]es arbres ilu- (sont) hauts pour moi, [ces hilu- (sont) hauts pour moi!]

$20^{\prime}$. Donc je suis monté, [...]»

$21^{\prime}$. [L'as]semblée [chante] avec eux [la même chose en réponse].

$22^{\prime}$. [...] chantent [ain]si: «Je suis allé [...].»

23'. (v. $3^{\text {ème }}$ pers. pl. inacc.). Les hommes d'Išstanuwa ...]

$24^{\prime}$. Derrière se tient la porte. [...]

$25^{\prime}-26^{\prime}$. Le roi, la reine, les prin[ces royaux ...] entrent. [...]

$27^{\prime}$. [...] dans le lieu [...]

$28^{\prime}$. [...] se tient. [...]

$29^{\prime} . \quad[\ldots]$ ne $[\ldots]$ pas

$30^{\prime} . \quad[\ldots] \ldots[\ldots]$

(lacune de 5 lignes env.)

$36^{\prime \prime}$. Tablette [...]

$37^{\prime \prime} . \quad$ de $[\ldots]$

$38^{\prime \prime} . \quad$ fils $[\ldots]$

$39^{\prime \prime}$ champ non cultivé $[\ldots]$

\section{Bibliographie}

Hutter, M. (2003): Aspects of Luwian Religion, dans: H. C. Melchert (éd.), The Luwians. HdO I/68. Leyde/Boston, 239-243.

Melchert, H. C. (1993): Cuneiform Luvian Lexicon. Chapel Hill.

Melchert, H. C. (2001): Cuneiform Luvian Corpus. Chapel Hill.

Starke, F. (1985): Die keilschrift-luwischen Texte in Umschrift. StBoT 30. Wiesbaden. 